\title{
The Application of Forensic Trace Evidence Analysis to the Detection, Identification and Sourcing of Alleged Foreign Matter in Pharmaceutical Products
}

\author{
Skip Palenik and Christopher S. Palenik
}

Microtrace LLC, Elgin, IL, USA

The identification and source determination of contaminants (or alleged contaminants) in pharmaceutical products has much in common with the forensic analysis of trace evidence that is encountered in the course of criminal investigations. Similar laboratory techniques are employed by both fields to detect, isolate, analyze and identify microscopic quantities of unknown substances or single small particles of interest. They differ primarily in their end uses: In the pharmaceutical industry, the primary goal is to locate the source of the identified foreign matter so that it can be eliminated from future lots of product, while in a criminal proceeding it is to provide clues to advance an investigation or to be presented as evidence at trial. As sensational reports of contaminated food and drugs have made the headlines in recent years, there is increased scrutiny of even innocuous foreign matter by the legal profession and litigious consumers. It has become of some interest, therefore, to perform laboratory analyses of foreign matter discovered in pharmaceutical products according to guidelines similar to those followed in the forensic science laboratory to prevent accusations that "proper procedures" were not followed, either by administrative agencies or by lawyers or courts. As a forensic trace evidence analysis laboratory that also conducts analysis of foreign matter for pharmaceutical companies, not only in North America but throughout much of the world, we have first-hand experience in the application to forensic methods of analysis to such problems.

In this presentation we will demonstrate, by means of examples from actual projects from our files, how alleged foreign matter in pharmaceuticals (both parenteral solutions, solid dosage forms, and laminates) may be located, isolated, analyzed and identified. It will also stress how seemingly minor procedures such as proper documentation, packaging, chain-of-custody, sample preservation, and ultimately the production of a self-contained narrative report are significant to the process and how they can be simultaneously implemented into the analysis process. The report as the final work product should contain sufficient supporting analytical data and observations documented by means of macro-photographs, spectra, chromatograms, photomicrographs and any other data that was obtained and relied upon to form the conclusions. Since such conclusion are, of necessity, at least partially subjective, the report must clearly state the reasoning used to arrive at it and how the data supports it. The clear separation of the facts (data and observations) and the interpretation of them that led to the conclusion(s) of identity, probable source, etc., must be clearly stated to minimize the possibility of misinterpretation.

Normally, detection (location) of contaminants presents little problems, since they are often obvious upon an initial inspection. For example, a white tablet shows colored spots, abnormally high particle counts are detected by an automated particle size instrument, or particles are seen floating in a drug solution in a vial. Occasionally, they are not so obvious and microscopical inspection, often assisted by atypical lighting geometries or alternate light sources (i.e., different wavelengths of light) is required to find them. 
Often more difficulty can be encountered in the isolation and preparation of these contaminants, especially when they occur in parenteral solutions. Isolation can often be effected by means of a "dipping tube," or sometimes a large drop containing the particle of interest can be removed with a Pasteur pipette. From these aliquots, the particles of interest can be placed on a microscope slide, removed, and washed. Drugs containing large protein molecules with very small contaminants are often processed by filtration through gold coated polycarbonate filters. The coating makes the substrate electrically conductive for morphological analysis in the SEM (by secondary electron imaging, backscatter imaging, elemental analysis by EDS) and reflective for double pass infrared microspectroscopy (to obtain chemical information). Such particles can also be probed by Raman microspectroscopy. Of course, the filter can also be examined at any stage under a stereomicroscope and individual foreign particles can be picked from the substrate in the clean room for whatever type of instrument or test is most likely to help identify it.

Because of its almost unlimited range of applicability in the hands of an experienced analytical microscopist, isolated particles are always examined first with the polarizing microscope (PLM) in an appropriate mounting medium. Substances as diverse as synthetic and natural fiber fragments, hairs, minerals, starches, pigments, drugs, chars, rubber, etc. can all be recognized and identified, to at least some degree, by means of their morphology and optical crystallographic properties. If more a more narrow identification is desired (which is of importance when comparison to suspected sources is possible and necessary), the knowledge of identity makes it possible to efficiently select the most appropriate test, instrument or observation. Thus, the identification of a particle as cellulose (by FTIR spectroscopy) may be helpful, but if it is desired to determine where it and the other fragments accompanying it have originated, it is possible to mount it in stains to study its microscopic morphology to decide if it is cotton, a bast fiber or a paper fiber. If paper, the pulping process can be determined and the microscopic anatomy of even fiber or tracheid fragments can be sufficient to determine the identity of the wood species from which the pulp was prepared. If cotton, the presence of an optical brightener can be determined by fluorescence microscopy, and colored fibers can be differentiated and compared using microsepctrofluorimetry with a microspectrophotometer. In a similar manner, adducts of protein and silicone, the latter of which is ubiquitous in vials and syringes today can be not only identified, but their distribution can be mapped by means of microchemical stains, infrared and Raman spectroscopy, or SEM/EDS.

The real world applications of the principles and approaches stated here will be demonstrated through examples of actual problems in both pharmaceutical and criminal forensic cases that have been submitted to us for investigation over the years. 